\title{
THE
}

\section{Carboxylesterase- 2 is a highly sensitive target of the antiobesity agent orlistat with profound implications in the activation of anticancer prodrugs}

\author{
Da Xiao \\ University of Rhode Island \\ Deshi Shi \\ University of Rhode Island \\ Dongfang Yang \\ University of Rhode Island \\ Benjamin Barthel
}

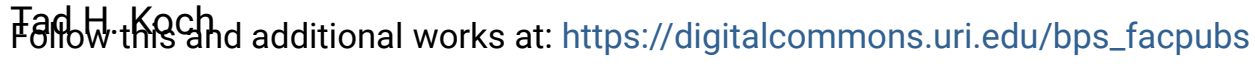

This is a pre-publication author manuscript of the final, published article.

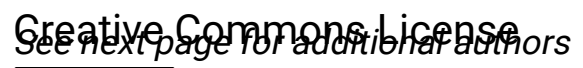

cc) (†) $\odot$

This work is licensed under a Creative Commons Attribution-Noncommercial-No Derivative Works 4.0 License.

\section{Citation/Publisher Attribution}

Xiao, D., Shi, D., Yang, D., Barthel, B., Koch, T. H., \& Yan, B. (2013). Carboxylesterase-2 is a highly sensitive target of the antiobesity agent orlistat with profound implications in the activation of anticancer prodrugs. Biochemical Pharmacology, 85(3), 439-447. doi: 10.1016/j.bcp.2012.11.026

Available at: https://doi.org/10.1016/j.bcp.2012.11.026

This Article is brought to you for free and open access by the Biomedical and Pharmaceutical Sciences at DigitalCommons@URI. It has been accepted for inclusion in Biomedical and Pharmaceutical Sciences Faculty Publications by an authorized administrator of DigitalCommons@URI. For more information, please contact digitalcommons-group@uri.edu. 


\section{Authors}

Da Xiao, Deshi Shi, Dongfang Yang, Benjamin Barthel, Tad H. Koch, and Bingfang Yan 


\title{
CARBOXYLESTERASE-2 IS A HIGHLY SENSITIVE TARGET OF THE ANTIOBESITY AGENT ORLISTAT WITH PROFOUND IMPLICATIONS IN THE ACTIVATION OF ANTICANCER PRODRUGS
}

\author{
Da Xiao ${ }^{1}$, Deshi Shi ${ }^{1}$, Dongfang Yang ${ }^{1}$, Benjamin Barthel' ${ }^{2}$, Tad H. Koch², and Bingfang \\ Yan ${ }^{1}$ \\ ${ }^{1}$ Department of Biomedical Sciences, Center for Pharmacogenomics and Molecular Therapy \\ University of Rhode Island, Kingston, RI 02881, USA \\ ${ }^{2}$ Department of Chemistry and Biochemistry University of Colorado, Boulder, CO 80309, USA
}

\begin{abstract}
Orlistat has been the most used anti-obesity drug and the mechanism of its action is to reduce lipid absorption by inhibiting gastrointestinal lipases. These enzymes, like carboxylesterases (CESs), structurally belong to the $\alpha / \beta$ hydrolase fold superfamily. Lipases and CESs are functionally related as well. Some CESs (e.g., human CES1) have been shown to hydrolyze lipids. This study was designed to test the hypothesis that orlistat inhibits CESs with higher potency toward CES1 than CES2, a carboxylesterase with little lipase activity. Liver microsomes and recombinant CESs were tested for the inhibition of the hydrolysis of standard substrates and the anticancer prodrugs pentyl carbamate of $p$-aminobenzyl carbamate of doxazolidine (PPD) and irinotecan. Contrary to the hypothesis, orlistat at $1 \mathrm{nM}$ inhibited CES2 activity by $75 \%$ but no inhibition on CES1, placing CES2 one of the most sensitive targets of orlistat. The inhibition varied among some CES2 polymorphic variants. Pretreatment with orlistat reduced the cell killing activity of PPD. Certain mouse but not rat CESs were also highly sensitive. CES2 is responsible for the hydrolysis of many common drugs and abundantly expressed in the gastrointestinal track and liver. Inhibition of this carboxylesterase probably presents a major source for altered therapeutic activity of these medicines if co-administered with orlistat. In addition, orlistat has been linked to various types of organ toxicities, and this study provides an alternative target potentially involved in these toxicological responses.
\end{abstract}

(C) 2012 Elsevier Inc. All rights reserved.

Address Correspondence to: Dr. Bingfang Yan, Department of Biomedical and Pharmaceutical Sciences, University of Rhode Island, Kingston, RI 02881, Phone: (401) 874-5032, Fax: (401) 874-5048, byan@uri.edu.

Publisher's Disclaimer: This is a PDF file of an unedited manuscript that has been accepted for publication. As a service to our customers we are providing this early version of the manuscript. The manuscript will undergo copyediting, typesetting, and review of the resulting proof before it is published in its final citable form. Please note that during the production process errors may be discovered which could affect the content, and all legal disclaimers that apply to the journal pertain.

The authors indicate no potential conflict of interest. 


\section{Keywords}

Orlistat; aspirin; irinotecan; liver; $\alpha / \beta$ fold hydrolases; carboxylesterases; first pass

\section{INTRODUCTION}

Obesity is probably the most important health issue and associated with a wide range of health conditions such as cardiovascular diseases, type II diabetes and certain types of cancers (1-3). In Great Britain, obesity crisis has risen 10 times during the past decade (4). In the United States (US), the direct cost of obesity is estimated as many as $10 \%$ of all medical cost (5). Among the US population, more than $30 \%$ are considered obese and almost $70 \%$ are overweight. The prevalence of obesity is slightly lower in men than women, but the prevalence in men has shown a clear trend of increase during the past decade. Importantly, obesity is no longer an issue of adults only, and the prevalence has reached an all-time high in children and adolescents (6), and more than $10 \%$ of these populations are considered obese or overweight. Overweight children have much higher chance of becoming obese adults.

Obesity and overweight are caused by a combination of excessive food intake and lack of exercise. Clearly, changes in life styles probably represent the most effective approach to lose weight (7). Intervention with medication has been increasingly used (8). Orlistat is widely used for this purpose and marketed under prescription and over-the-counter (9). The mechanism of action is to inhibit pancreatic and functionally related lipases. Pancreatic lipases are normally secreted into the lumen of the small intestine and hydrolyze triglycerides (11). Lipids are absorbed upon hydrolysis, and inhibition of the lipases reduces the hydrolysis, thus decreasing the fat intake. In addition to weight loss, orlistat has been shown to improve conditions such as hypertension and type II diabetes (12). On the other hand, orlistat has been implicated with severe liver toxicity (13).

Lipases, like carboxylesterases (CESs), structurally belong to the $\alpha / \beta$ fold hydrolase superfamily (10). While CESs hydrolyze drugs and other xenobiotics, some CESs hydrolyze lipids as well $(14,15)$. In the human genome, seven CES genes exist with one being a pseudogene $(16,17)$. Nonetheless, only three human CESs are catalytically characterized: CES1, CES2 and CES3 (17). CES3 is much weaker enzyme in terms of metabolizing common drugs. In a proteomic study, CES1 was identified as one of the top ten most abundant proteins in the adult liver (18). More importantly, CES1 but not CES2 has been shown to hydrolyze neutral lipids (15). In contrast, CES2 preferably hydrolyzes bulky molecules such as the anticancer agents: irinotecan (19) and pentyl carbamate of $p$ aminobenzyl carbamate of doxazolidine (PPD) (20, 21).

This study was performed to test the hypothesis that orlistat inhibits CESs with higher potency toward CES1 than CES2, a carboxylesterase with little lipase activity. To test this hypothesis, recombinant CESs from human, mouse and rat were incubated with orlistat at various concentrations (1-100 $\mathrm{nM}$ ) and the hydrolytic activity was determined. While orlistat inhibited all CESs tested, however, the relative potency varied markedly. Contrary to the hypothesis, CES2 was much more sensitive than CES1. Among all CESs, human CES2 
and mouse ces $2 \mathrm{c}$ were inhibited to the most extent and represent one of the most sensitive targets of orlistat. The inhibition was irreversible and occurred intracellularly. Orlistat inhibited the activation of PPD and irinotecan, thus decreasing its cell killing activity.

\section{MATERIALS AND METHODS}

\subsection{Chemicals and supplies}

Cycloheximide (CHX), p-nitrophenylacetate, 1-naphthylacetate, orlistat and Hanks balanced salt solution were from Sigma (St. Louis, MO). Dulbecco's modified eagle medium (DMEM) and high fidelity Platinum Taq DNA polymerase were from Life Technologies (Carlsbad, CA). The antibody against glyceradehyde-3-phosphate dehydrogenase (GAPDH) was from Abcam (Cambridge, UK). The goat anti-rabbit IgG conjugated with horseradish peroxidase was from Pierce (Rockford, IL). Nitrocellulose membranes were from Bio-Rad (Hercules, CA). Expression constructs for mouse CESs were purchased from OriGene (Rockville, MD). Synthesis of PPD was described elsewhere (20, 21). Unless otherwise specified, all other reagents were purchased from Thermo-Fisher Scientific (Fair Lawn, NJ).

\subsection{Enzymatic assays}

Liver tissues were homogenized and microsomes were prepared by differential centrifugation as described previously (22-24). Human liver tissues $(n=14$, equal number of each gender) were from the University of Maryland Brain and Tissue Bank for Developmental Disorders (Baltimore, MD) (24). Sprague-Dawley rats (male, $n=4$ ) and CD-1 mice (male, $\mathrm{n}=4$ ) were from Charles River (Wilmington, MA). The use of the human samples and animals was approved by the Institutional Review Board and the Institutional Animal Care and Use Committee. The enzymatic activity was determined spectrophotometrically as described previously (19). The activity was also determined by native gel electrophoresis stained for carboxylesterase activity (25). Briefly, liver homogenates $(10 \mu \mathrm{g})$ were solubilized with $0.2 \%$ Lubrol and subjected to electrophoresis through a $3 \%$ acrylamide stacking gel and a $7.5 \%$ acrylamide separating gel. After electrophoresis, the gels were washed for $1 \mathrm{~h}$ in $100 \mathrm{mM}$ potassium phosphate buffer $(\mathrm{pH}$ 6.5), followed by incubating in the same buffer containing 1-naphthylacetate (5 mM) and 4benzolamino-2,5-dimethoxybenzenediazonium chloride hemi (zinc chloride) salt, usually termed Fast Blue RR $(0.4 \mathrm{mg} / \mathrm{ml})$. Staining for carboxylesterases by this method is based on the formation of a black, insoluble complex between the hydrolytic products and Fast Blue RR. The images were captured by Carestream 2200 PRO Imager.

\subsection{Western analysis}

Samples were resolved by 7.5\% SDS-PAGE in a mini-gel apparatus and transferred electrophoretically to nitrocellulose membranes. After non-specific binding sites were blocked with 5\% non-fat milk, the blots were incubated with an antibody against CES1, CES2 or GAPDH. The antibodies against CES1 and CES2 were prepared with synthetic peptides and purified as described previously $(24,26)$. The specificity was established with the corresponding recombinant proteins. The primary antibodies were localized with goat anti-rabbit IgG conjugated with horseradish peroxidase. Horseradish peroxidase activity was 
detected with a chemiluminescent kit (SuperSignal West Pico). The chemiluminescent signal was captured by Carestream 2200 PRO Imager.

\subsection{Site-directed mutagenesis and cell transfection}

There are several polymorphic variants reported in the literature or in the database of the National Center for Biotechnology Information. To shed light on the sensitivity to orlistat, variants encoding single mutation were prepared by site-directed mutagenesis as described previously (27). The parent CES2 plasmid was isolated with a cDNA trapping method and reported previously (26). Complementary oligonucleotides (Table I) were synthesized, annealed to the CES2 expression construct, and subjected to a thermocycler for a total of 15 cycles. The resultant PCR-amplified constructs were then digested with Dpnl to remove the nonmutated parent construct. The mutated PCR-amplified constructs were used to transform XL1-Blue. All mutated constructs were subjected to sequencing analysis to confirm the desired mutation being made without secondary mutations. To prepare the corresponding recombinant CES2 variant, 293T cells were transfected with the wild-type or a mutant construct with Genjet version II from SignaGen Lab (Rockville, MD) as described by the manufacturer. The transfected cells were harvested after a-24 incubation and cell lysates were prepared. Seven mutants were made including N85Q, A139T, A178V, R180H, A187P, G223R or N250Q (single amino acid substitution).

\subsection{Cell viability assay}

Cells (HepG2 and LS180) were seeded into 96-well plates (10,000/well). In some cases, cells were seeded in 6-well plates and transfected with CES2 or the vector. The transfected cells were then seeded in 96-well plates $24 \mathrm{~h}$ after the transfection. The seeded cells, usually overnight after seeding, were treated with one or more chemicals and cultured in normal medium (chemicals kept the same) for $48 \mathrm{~h}$. Thereafter, the medium was replaced with fresh medium containing MTT [(4,5-dimethylthiazolyl-2)-2,5-diphenyltetrazolium bromide] at a final concentration of $0.5 \mathrm{mg} / \mathrm{ml}$. After a $2-\mathrm{h}$ incubation at $37^{\circ} \mathrm{C}$, the medium was gently decanted, and DMSO (100 $\mu \mathrm{l} /$ well) was added to dissolve formazan product. The optical density (OD) was determined at $570 \mathrm{~nm}$, and the final OD values were calculated by subtracting the background reading (no seeded cells).

\subsection{Molecular modeling}

To gain molecular insight regarding orlistat-inhibition of CESs, we performed molecular modeling and docking studies. The structure of CES2 was modeled with Discovery Studio based on the crystal structure of CES1 (www.pdb.org). CES1 structure was retrieved from the Protein Data Bank (www.pdb.org). Water molecules were removed, and hydrogen atoms were added if they were missing in the original structural file. Standard protonation states of the residues were adopted to produce the charged acidic and basic side chains as well as the $\mathrm{N}$ - and C-termini. The homology modeling of CES2 was also performed with Discovery Studio-client based on sequence similarity and the homology/analogy recognition engine Phyre (28). The 3-D model of CES2 was evaluated with the "Verify Protein (Profiles-3D)". The modeled CES2 structure was tested for the interaction with orlistat with Autodock 4.2. To compare CES1 with CES2 for the residues in interacting with orlistat, two major 
sequences of CES2 were replaced with the corresponding sequences of CES1. The subsequent protein mutant was analyzed for the changes in the interaction with orlistat. Once again, the 3-D structure was constructed with Phyre but the interaction was determined with Autodock 4.2.

\subsection{Statistical analysis}

Data are presented as mean \pm SD of at least three separate experiments, except where results of blots are shown in which case a representative experiment is depicted in the figures.

Statistical significance between two means was made by One-way ANOVA followed by a DUNCAN's multiple comparison test $(p<0.05)$. Asterisks or lines were used to indicate data-points for the comparisons.

\section{RESULTS}

\subsection{Orlistat is a potent inhibitor of human and mouse but not rat CESs}

Orlistat targets gastrointestinal lipases with high selectivity (29). However, clinical observations suggest that this drug acts on other targets that may confer beneficial or adverse effects (13). In this study, we made an effort to determine whether orlistat inhibits CESs, a class of enzymes that are structurally related to lipases. Pooled liver microsomes from humans, mice or rats were incubated with orlistat at various concentrations $(0-1000$ $\mathrm{nM}$ ) and tested for hydrolytic activity. As shown in Fig. 1A, orlistat at $1 \mathrm{nM}$ significantly inhibited the hydrolysis in human microsomes and $~ 50 \%$ inhibition was detected at $50 \mathrm{nM}$ orlistat. Increased concentrations up to $1000 \mathrm{nM}$ further increased the inhibition but the increase was moderate ( $20 \%)$. Similar concentration-dependent inhibition was detected with mouse microsomes (Fig. 1B). Surprisingly, orlistat was much less potent in the inhibition of the hydrolysis in rat microsomes (Fig. 1C). A $10 \%$ inhibition was detected at $50 \mathrm{nM}$ and $30 \%$ at $1000 \mathrm{nM}$. Overall, the inhibition in rat microsomes was less than half of that in human or mouse counterparts (Fig. 1).

\subsection{Differential inhibition among CESs}

It was unexpected that mouse microsomes shared with human but not rat microsomes in terms of the inhibition profile, although mouse and rat share more similarities in terms of the tissue distribution of CESs and the number of CES genes (17). We did not have all human and rodent CESs in purified form or in expression construct. Nonetheless, we tested whether the inhibition varies among CESs by native gel electrophoresis stained for hydrolytic activity (25). This experiment took advantage of the differences of CESs in electrophoretic mobility and the observation that many CESs are catalytically active in polyacrylamide gel. 1-Naphthylacetate was used as the substrate, although not all CESs hydrolyze this ester to similar extent. As shown in Fig. 2A, two dominant activity bands were detected in human liver microsomes. Importantly, only the lower band (CES2) was profoundly reduced upon incubation with orlistat. As expected, multiple bands were detected with mouse microsomes, and two of them (ces2c and ces2e) were inhibited by orlistat (Fig. 2B). Rat microsomes produced three major and three minor bands, but none of them were evidently inhibited (Fig. 2C). It should also be emphasized that the identity of carboxylesterases was established based on recombinant carboxylesterases (25). In summary, human CES2 and mouse ces2c 
and ces2e were highly sensitive to orlistat but human CES1, mouse ces1d, rat ces1d and ces1e were relatively resistant. It should be emphasized that the inhibition detected by this method with the removal of orlistat by electrophoresis established that orlistat is an irreversible inhibitor of these CESs.

\subsection{Super-sensitivity of human CES2, mouse ces2c and ces2e to orlistat}

The staining experiment provided initial information on the profound difference among various carboxylesterases toward orlistat inhibition. To confirm this finding, recombinant CESs were produced and tested for their sensitivity to orlistat. As shown in Fig. 3A, human CES2 but not CES1 was highly sensitive to orlistat inhibition. Orlistat at $1 \mathrm{nM}$ inhibited CES2 by 75\%, but no inhibition was detected with CES1. Even at $100 \mathrm{nM}$ orlistat, CES1 was inhibited by $\sim 30 \%$ only (Fig. 3A). Like CES2, mouse ces $2 \mathrm{c}$ and ces2e were potently inhibited by $1 \mathrm{nM}$ orlistat: namely 90 and 55\%, respectively. In contrast, ces1d was inhibited by $12 \%$ only at this concentration (Fig. 3B). In a striking contrast, rat ces1d and ces1e, two major forms of liver CESs, were resistant to orlistat. Even at $10 \mathrm{nM}$, only a $20 \%$ inhibition was detected for both enzymes (Fig. 3C). In summary, human CES2, mouse ces2c and ces2e were highly sensitive but human CES1, mouse ces1d, rat ces1d and ces1e were relatively resistant.

\subsection{Cellular inhibition as a function of time}

We next tested whether the inhibition of CES2 takes place within cells. This was of significance as orlistat is generally considered to be absorbed poorly. To test this possibility, LS180 cells (a colon adenocarcinoma line) were treated with orlistat for 1-24 h, washed extensively and lysed by sonication. The lysates were then tested for the hydrolytic activity. As shown in Fig. 4A, the activity of CES2 but not CES1 was inhibited by $~ 80 \%$ within1-h incubation. Comparable inhibition was detected when cells were treated for $6 \mathrm{~h}$. Interestingly, prolonged inhibition (i.e., $24 \mathrm{~h}$ ) was less effective. We next tested whether the less inhibition by prolonged incubation was due to increased expression of CES2. Western blotting was performed with the same gel stained for activity. As predicated, prolonged incubation increased CES2 expression (Fig. 4A), suggesting that orlistat was depleted by prolonged incubation, presumably by interacting with other enzymes. To further confirm this possibility, LS180 cells were treated with orlistat, CHX (a protein synthesis inhibitor) or both for $24 \mathrm{~h}$, and the activity of CES2 was determined. As shown in Fig. 4B (Top), orlistat, CHX and co-treatment (both) all markedly inhibited CES2 with the co-treatment causing the maximal inhibition. Western blotting detected decreases in CES2 expression in cells treated with CHX (Middle of Fig. 4B).

\subsection{Reversal of PPD mediated cell-killing by orlistat}

Next, we tested whether the inhibition has any clinical significance. Cells were treated with PPD in the presence or absence of orlistat. PPD is a carbamate anticancer prodrug and activated preferentially by CES2 hydrolysis of the ester side chain of the carbamate $(20,21)$. As shown in Figs. 5A and B, treatment with orlistat alone caused no changes in cell viability, whereas treatment with PPD alone caused significant reduction in cell viability in both LS180 and HepG2 lines. The reduction, however, was significantly reversed by orlistat 
in both cell lines. Fig. 5C shows the representative image of growth status of cells treated with orlistat, PPD or both.

\subsection{Differential inhibition of CES2 polymorphic variants}

Our previous study suggested that CES2-activity varied markedly among individual livers (30). We next tested whether orlistat-inhibition exhibits large individual variations as well. Individual liver samples were incubated with orlistat and then the remaining hydrolytic activity was determined. As shown in Fig. $6 \mathrm{~A}$, all samples were inhibited by $\sim 15 \%$ with a few exceptions (Fig. 6A). Samples 4 and 5 were inhibited by less than $10 \%$ whereas samples 7 and 14 by 40 and $21 \%$, respectively. The individual differences pointed to the existence of polymorphic variants that differ in responding to orlistat. To test this possibility, we tested several CES2 variants for orlistat inhibition. As shown in Fig. 6B, variants A139T and A178V were relatively resistant, whereas the variant $\mathrm{R} 180 \mathrm{H}$ was relatively more sensitive, particularly when orlistat was used at $1 \mathrm{nM}$. The relative sensitivity among these variants was confirmed by the native gel staining method (Fig. 6C). The lysates from CES2transfected cells exhibited much greater inhibition than human liver microsomes (Figs. 6A and $\mathrm{B}$ ), even when the same substrate ( $p$-nitrophenylacetate) was used. The reason was that the microsomes but not the lysates contained CES1, which rapidly hydrolyzed $p$ nitrophenylacetate but was resistant to orlistat. We next tested whether the substitutions of these residues participate in direct contact with orlistat. Molecular docking was performed. As shown in Fig. 6D, approximately 20 residues interact directly with orlistat. However, none of them were those identified to support altered sensitivity to orlistat (i.e., A139T, A178V and R180H).

\subsection{Correlation between PPD and irinotecan activation by CES2 variants}

Irinotecan, like PPD, is predominately activated by CES2 $(31,32)$. We next tested whether these variants activate irinotecan and PPD to a similar extent. To link the hydrolysis directly to anticancer activity, cells were transfected to express CES2 or a variant and then treated with PPD $(0.1 \mu \mathrm{M})$, irinotecan $(1 \mu \mathrm{M})$ or the solvent. Cell viability was determined and the results were calculated as the percentage of the treatment with solvent control. As shown in Fig. 7A, cell viability with irinotecan was generally higher than that with PPD, although irinotecan was tested at higher concentration, suggesting that irinotecan was less potent. Interestingly, variants such as A139T and A178V comparably activated irinotecan but differed markedly in activating PPD. Overall, hydrolytic activation of irinotecan correlated moderately with that of PPD $(r=0.6731)$ (Right of Fig. 7A). These results suggest that these two esters, although predominately activated by CES2, differ somewhat in interacting with CES2.

\section{DISCUSSION}

For more than a decade, orlistat has been a major anti-obesity drug (9). In addition to weight loss, orlistat is shown to improve metabolic syndrome in general (12). In other cases, orlistat has been linked to certain organ toxicity as well (13). The molecular targets responsible for the organ toxicity remain to be determined. In this study, we have characterized a novel target of orlistat: CES2, a major enzyme in drug metabolism and detoxication of xenobiotics 
(21). Inhibition of CES2 by orlistat occurred intracellularly and decreased the hydrolytic activation of anticancer drugs. Based on the concentration-inhibition curve, CES2 was even more sensitive than lipases. Orlistat at $2 \mathrm{nM}$ inhibited porcine pancreatic lipase by $41 \%$ (29) but caused a 70\% inhibition of CES2 (Fig. 3). Fatty acid synthase, another orlistat target, is relatively resistant. Based on substrate labeling study, micromolar concentrations of orlistat were needed to effectively inhibit this synthase (33). In humans after oral administration at therapeutic doses, orlistat reaches a blood concentration of $0.20-8.77 \mathrm{ng} / \mathrm{ml}$ depending on an individual (34). Its major metabolites, on the other hand, have concentrations of 20-50 times of the parent compound $(34,35)$. It is therefore estimated that orlistat can reach lowmicromolar concentrations after oral administration in the mucosa of the gastrointestinal track and the liver. Clearly, CES2 can be potently inhibited by regular dosing regimens of orlistat.

It was interesting to notice that lipolytic activity of a CES was not a contributing factor to the potent inhibition of orlistat. Human CES1, rat ces1d and mouse ces1d were all known to have lipolytic activity $(14,15)$, but surprisingly inhibited to a much lesser extent by orlistat (Fig. 3). Similar observation was made on a porcine liver triacylglycerol hydrolase (36) and in rat primary hepatocytes (37). It can not be excluded, however, that orlistat efficaciously inhibits these CESs in the presence of a lipid/water interface. Orlistat reportedly inhibited lipoprotein lipase in the presence but not in the absence of the interface (38). In this study, however, we used cell-based assays (mimicking closely to therapeutic situation) and detected portent inhibition of CES2 but not CES1 (Fig. 4), demonstrating that CES2 is a more sensitive target than CES1 even in the native condition.

Based on the relative sensitivity toward orlistat, CESs can be divided into major types: highly sensitive type such as CES2 and the other relatively resistant type such as CES1. In human liver microsomes, a $46 \%$ inhibition was detected when orlistat was used at $50 \mathrm{nM}$, but 20 times this concentration $(1 \mu \mathrm{M})$ caused only a $20 \%$ additional inhibition (Fig. 1A). Similar observation was made with mouse microsomes (Fig. 1B). Rat CESs, on the other hand, were less sensitive, consistent with the report using primary hepatocytes (37). Orlistat at $50 \mathrm{nM}$ caused a $10 \%$ inhibition and 30\% inhibition at $1 \mu \mathrm{M}$ orlistat (Fig. 1C). Overall, rat CESs are much less sensitive than human and mouse CESs. These findings suggest that mouse is a better model for humans on orlistat-inhibition. In addition to species difference, there were individual variations as well. Certain individuals exhibited more than four times the difference in orlistat inhibition (sample 4 versus 7) (Fig. 6B).

Genetic polymorphism of CES2 is likely a major contributor to the individual variation in orlistat inhibition. In this study, we have shown that CES2 variants such as A139T and A178V were resistant to orlistat, whereas R180H was more sensitive (Fig. 6B). Interestingly, none of these residues directly line up with the catalytic cavity based on the docking study (Fig. 6B). On the other hand, sequence alignment has revealed three regions where the highly sensitive CESs share with or differ markedly from less sensitive CESs. These residues differ in the charges or lipophilicity (Fig. 7B). One of the regions (58-93 amino acids) harbors the helix $a 1$, which is part of the catalytic domain (39). The region (256-280 amino acid), part of the $\alpha \beta$ domain, has a 15 amino acid deletion among sensitive 
CESs. The region from residue 390 to 428 stretches helices a 13 to a 14 . Like the helix a 1 , these two helices are parts of the catalytic domain.

Orlistat-inhibition on CESs may have profound clinical significance. CES2 is a major CES that is highly expressed in the gastrointestinal track and liver. Many ester drugs such as aspirin and prasugrel are hydrolyzed primarily by CES2 $(30,40)$. Both aspirin and prasugrel are antiplatelet agents. However, hydrolysis has opposite effect on the therapeutic potential of these drugs. Hydrolysis of aspirin decreases the therapeutic activity, whereas hydrolysis is required for prasugrel to gain therapeutic activity. As a result, inhibition of CES2 by orlistat would enhance the antiplatelet activity of aspirin, but the opposite would be true for prasugrel. Aspirin is one of the oldest drugs but prasugrel is a new drug. Interestingly, aspirin-orlistat interactions have not been well characterized. One of major adverse effects of orlistat is oily spotting (13). It is likely that such a gastrointestinal side effect may actually decrease the absorption of aspirin, thus minimizing otherwise evident orlistat-aspirin interaction. Nevertheless, we have shown that co-treatment with orlistat significantly reversed the cell killing activity of PPD (Fig. 5), a prodrug that is hydrolytically activated by CES2 (20, 21).

In summary, our work points to several important conclusions. Firstly, we have shown that orlistat is a potent inhibitor of several major human and mouse CESs, establishing that orlistat decreases the hydrolysis of not only lipophilic but also hydrophilic compounds. Secondly, several polymorphic variants of CES2 have been found to differ markedly from the wild-type enzyme in orlistat inhibition, pointing to a possibility of large individual variation. Thirdly, inhibition of CES2 by orlistat significantly decreases the cell killing activity of PPD, suggesting that co-administration of orlistat alters the therapeutic potentials of drugs activated or eliminated by CES2 (41). CES2 is a major detoxication enzyme in the gastrointestinal track and in the liver. Orlistat has been linked to various types of organ toxicities. Characterization of CES2 as a novel orlistat target provides an alternative explanation to the organ-based toxicity.

\section{Acknowledgments}

This work was supported by NIH grants R01GM61988 and R01ES07965 to BY and R21CA143549-01A1 to THK. Further support to THK came from the Colorado Center for Drug Discovery.

\section{Abbreviation}

$\begin{array}{ll}\text { CES } & \text { carboxylesterase } \\ \text { CHX } & \text { cycloheximide } \\ \text { DMEM } & \text { Dulbecco's modified eagle medium } \\ \text { GAPDH } & \text { glyceraldehyde-3-phosphate dehydrogenase } \\ \text { PPD } & \text { pentyl carbamate of } p \text {-aminobenzyl carbamate of doxazolidine }\end{array}$




\section{References}

1. Bornfeldt KE, Tabas I. Insulin resistance, hyperglycemia, and atherosclerosis. Cell Metab. 2011; 14:575-585. [PubMed: 22055501]

2. Morselli LL, Guyon A, Spiegel K. Sleep and metabolic function. Pflugers Arch. 2012; 463:139_ 160. [PubMed: 22101912]

3. Shimizu M, Kubota M, Tanaka T, Moriwaki H. Nutraceutical approach for preventing obesityrelated colorectal and liver carcinogenesis. Int J Mol Sci. 2012; 13:579-95. [PubMed: 22312273]

4. 10,000 treated in hospital for being dangerously fat as admissions rise TENFOLD in a decade 2011. http://www.dailymail.co.uk/health/article-1360210/Obesity-crisis-Hospitals-treat-10-000-patientsdangerously-fat.html\#ixzz1p6Ux7QNJ

5. Reinberg, S. 2011. http://abcnews.go.com/Health/Healthday/story?id=8184975\&page $=1 \#$. T2CzwoEpZK0

6. Ogden CL, Carroll MD, Kit BK, Flegal KM. Prevalence of obesity and trends in body mass index among US children and adolescents in 1999-2010. JAMA. 2012; 307:483-90. [PubMed: 22253364]

7. Moyad MA. The optimal male health diet and dietary supplement program. Urol Clin North Am. 2012; 39:89-107. [PubMed: 22118348]

8. Powell AG, Apovian CM, Aronne LJ. New drug targets for the treatment of obesity. Clin Pharmacol Ther. 2011; 90:40-51. [PubMed: 21654742]

9. Kang JG, Park CY. Anti-Obesity Drugs: A Review about Their Effects and Safety. Diabetes Metab J. 2012; 36:13-25. [PubMed: 22363917]

10. Jochens H, Hesseler M, Stiba K, Padhi SK, Kazlauskas RJ, Bornscheuer UT. Protein engineering of $\alpha / \beta$-hydrolase fold enzymes. Chembiochem. 2011; 12:1508-1517. [PubMed: 21506229]

11. Bhutani MS, Gupta V, Guha S, Gheonea DI, Saftoiu A. Pancreatic cyst fluid analysis-a review. J Gastrointestin Liver Dis. 2011; 20:175-180. [PubMed: 21725515]

12. Coutinho W. The first decade of sibutramine and orlistat: a reappraisal of their expanding roles in the treatment of obesity and associated conditions. Arq Bras Endocrinol Metabol. 2009; 53:26270. [PubMed: 19466219]

13. Filippatos TD, Derdemezis CS, Gazi IF, Nakou ES, Mikhailidis DP, Elisaf MS. Orlistat-associated adverse effects and drug interactions: a critical review. Drug Saf. 2008; 31:53-65. [PubMed: 18095746]

14. Lehner R, Vance DE. Cloning and expression of a cDNA encoding a hepatic microsomal lipase that mobilizes stored triacylglycerol. Biochem J. 1999; 343:1-10. [PubMed: 10493905]

15. Zhao B, Song J, Ghosh S. Hepatic overexpression of cholesteryl ester hydrolase enhances cholesterol elimination and in vivo reverse cholesterol transport. J Lipid Res. 2008; 49:2212-7. [PubMed: 18599737]

16. Yan B, Matoney L, Yang D. Human carboxylesterases in term placentae: enzymatic characterization, molecular cloning, and evidence for the existence of multiple forms. Placenta. 1999; 20:599-607. [PubMed: 10452915]

17. Yan, B. Hydrolytic Enzymes. In: Zanger; Anzenbacher, editors. Metabolism of Drugs and Other Xenobiotics. WILEY-VCH Verlag GmbH \& Co. KGaA; Weinheim: 2012. p. 165-199.

18. Sun A, Jiang Y, Wang X, Liu Q, Zhong F, He Q, Guan W. Liverbase: a comprehensive view of human liver biology. J Proteome Res. 2010; 9:50-58. [PubMed: 19670857]

19. Xie M, Yang D, Wu M, Xue B, Yan B. Mouse liver and kidney carboxylesterase (M-LK) rapidly hydrolyzes antitumor prodrug irinotecan and the $\mathrm{N}$-terminal three quarter sequence determines substrate selectivity. Drug Metab Dispos. 2003; 31:21-27. [PubMed: 12485949]

20. Barthel BL, Torres RC, Hyatt JL, Edwards CC, Hatfield MJ, Potter PM, Koch TH. Identification of human intestinal carboxylesterase as the primary enzyme for activation of a doxazolidine carbamate prodrug. J Med Chem. 2008; 51:298-304. [PubMed: 18173233]

21. Barthel BL, Zhang Z, Rudnicki DL, Coldren CD, Polinkovsky M, Sun H, Koch GG, Chan DC, Koch TH. Preclinical efficacy of a carboxylesterase 2-activated prodrug of doxazolidine. J Med Chem. 2009; 52:7678-7688. [PubMed: 19634903] 
22. Yang J, Yan B. Photochemotherapeutic agent 8-methoxypsoralen induces the expression of cytochrome P450 3A4 and carboxylesterase HCE2: evidence on a differential involvement of the pregnane X receptor. Toxicol Sci. 2007; 95:13-22. [PubMed: 17003103]

23. Wyde ME, Kirwan SE, Zhang F, Laughter A, Hoffman HB, Bartolucci-Page E, Gaido KW, Yan B, You L. Di-n-butyl phthalate activates constitutive androstane receptor and pregnane $\mathrm{X}$ receptor and enhances the expression of steroid-metabolizing enzymes in the liver of rat fetuses. Toxicol Sci. 2005; 86:281-290. [PubMed: 15901914]

24. Shi D, Yang D, Prinssen EP, Brian E, Davies BE, Yan B. Surge in expression of carboxylesterase-1 during the post-natal stage enables a rapid gain of the capacity to activate the anti-influenza prodrug oseltamivir. J Infect Dis. 2011; 203:937-942. [PubMed: 21402544]

25. Xiao D, Yi-Tzai Chen, Yang D, Yan B. Age-related inducibility of carboxylesterases by antiepileptic agent phenobarbital and implication in drug metabolism and lipid accumulation. Biochem Pharmacol. 2012; 84:232-239. [PubMed: 22513142]

26. Zhu W, Song L, Zhang H, Matoney L, LeCluyse E, Yan B. Dexamethasone differentially regulates the expression of carboxylesterase genes in humans and rats. Drug Metab Dispos. 2000; 28:186191. [PubMed: 10640517]

27. Liu F, Yang D, Song X, Deng R, Yan B. The far and distal enhancers in the CYP3A4 gene coordinates the proximal promoter in responding to the pregnane $\mathrm{X}$ receptor similarly but differentially to hepatocyte nuclear factor-4a. Biochem J. 2008; 409:243-250. [PubMed: 17764444]

28. Vistoli G, Pedretti A, Mazzolari A, Testa B. Homology modeling and metabolism prediction of human carboxylesterase-2 using docking analyses by GriDock: a parallelized tool based on AutoDock 4.0. J Comput Aided Mol Des. 2010; 24:771-787. [PubMed: 20623318]

29. Hadváry P, Sidler W, Meister W, Vetter W, Wolfer H. The lipase inhibitor tetrahydrolipstatin binds covalently to the putative active site serine of pancreatic lipase. J Biol Chem. 1991; 266:2021-2027. [PubMed: 1899234]

30. Tang M, Mukundan M, Yang J, Charpentier N, LeCluyse EL, Black C, Yang D, Yan B. Antiplatelet agents aspirin and clopidogrel are hydrolyzed by distinct carboxylesterases and clopidogrel is transesterificated in the presence of ethyl alcohol. J Pharmacol Exp Ther. 2006; 319:1467-1476. [PubMed: 16943252]

31. Wu MH, Yan B, Humerickhouse R, Dolan ME. Irinotecan activation by human carboxylesterases in colorectal adenocarcinoma cells. Clin Cancer Res. 2002; 8:2696-2700. [PubMed: 12171903]

32. Yang J, Shi D, Yang D, Song X, Yan B. Interleukin-6 suppresses the expression of carboxylesterases HCE1 and HCE2 through transcriptional repression. Mol Pharmacol. 2007; 72:686-694. [PubMed: 17537833]

33. Kridel SJ, Axelrod F, Rozenkrantz N, Smith JW. Orlistat is a novel inhibitor of fatty acid synthase with antitumor activity. Cancer Res. 2004; 64:2070-2075. [PubMed: 15026345]

34. Zhi J, Melia AT, Funk C, Viger-Chougnet A, Hopfgartner G, Lausecker B, Wang K. Metabolic profiles of minimally absorbed orlistat in obese/overweight volunteers. J Clin Pharmacol. 1996; 36:1006-1011. [PubMed: 8973989]

35. Zhi J, Mulligan TE, Hauptman JB. Long-term systemic exposure of orlistat, a lipase inhibitor, and its metabolites in obese patients. J Clin Pharmacol. 1999; 39:41-46. [PubMed: 9987699]

36. Lehner R, Verger R. Purification and characterization of a porcine liver microsomal triacylglycerol hydrolase. Biochemistry. 1997; 36:1861-1868. [PubMed: 9048571]

37. Hermier D, Hales P, Brindley DN. Effects of the lipase inhibitors, Triton WR-1339 and tetrahydrolipstatin, on the synthesis and secretion of lipids by rat hepatocytes. FEBS Lett. 1991; 286:186-188. [PubMed: 1864367]

38. Lookene A, Skottova N, Olivecrona G. Interactions of lipoprotein lipase with the active-site inhibitor tetrahydrolipstatin (Orlistat). Eur J Biochem. 1994; 222:395-403. [PubMed: 8020477]

39. Bencharit S, Morton CL, Hyatt JL, Kuhn P, Danks MK, Potter PM, Redinbo MR. Crystal structure of human carboxylesterase 1 complexed with the Alzheimer's drug tacrine: from binding promiscuity to selective inhibition. Chem Biol. 2003; 10:341-349. [PubMed: 12725862]

40. Laizure SC, Parker RB. A comparison of the metabolism of clopidogrel and prasugrel. Expert Opin Drug Metab Toxicol. 2010; 6:1417-1424. [PubMed: 20839914] 
41. Xiao D, Yang D, Charpentier M, Yan B. Regulation of carboxylesterase-2 expression by p53 family proteins and enhanced anticancer activities among 5-fluorouracil, irinotecan and doxazolidine prodrug. Brit J Pharmacol. 2012 In revision. 


\section{A. Human}

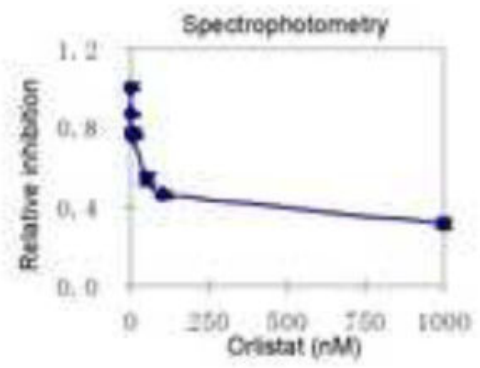

B. Mouse

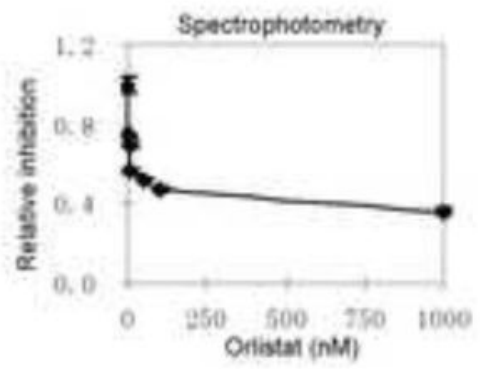

C. Rat

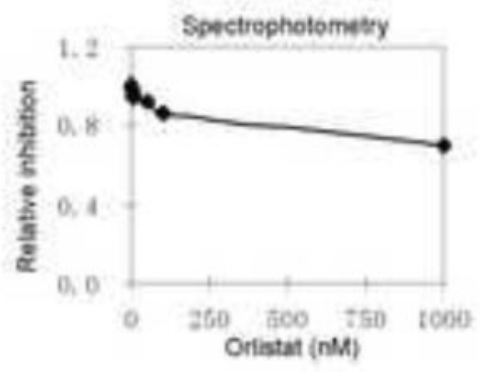

Fig. 1. Inhibition of $p$-nitrophenylacetate hydrolysis of pooled microsomes from human (A), mouse (B) or rat (C) livers by orlistat

Microsomes $(15 \mu \mathrm{g})$ from human liver $(n=14)$, mouse liver $(n=4)$ or rat liver $(n=4)$ were pre-incubated in a total volume of $990 \mu \mathrm{l}$ with orlistat at various concentrations $(0-1 \mu \mathrm{M})$ for $30 \mathrm{~min}$. The pre-incubated mix was then transferred into sample cuvette $(1 \mathrm{ml})$ in $100 \mathrm{mM}$ potassium phosphate buffer, $\mathrm{pH}$ 7.4. Reactions were initiated by adding $p$-nitrophenylacetate ( $10 \mu \mathrm{l}$ of $100 \mathrm{mM}$ stock in acetonitrile) and hydrolytic rate was recorded from an increase in absorbance at $400 \mathrm{~nm}$. The absorbance without orlistat was expressed as 1.0. Results were from three separate experiments. 


\section{A. Human}

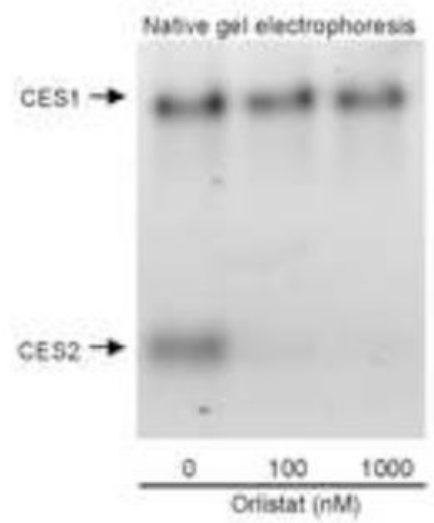

B. Mouse

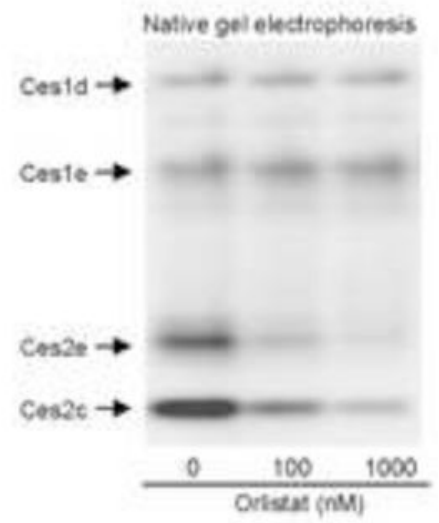

C. Rat

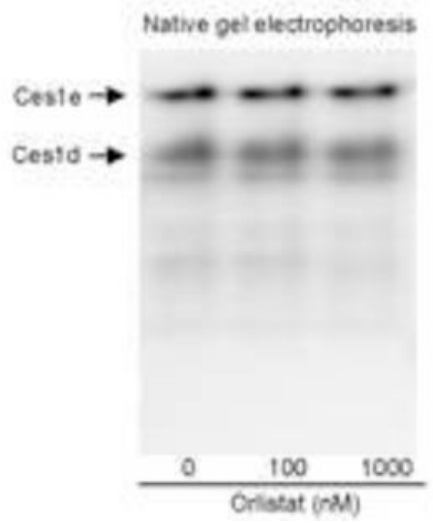

Fig. 2. Non-denaturing electrophoresis stained for hydrolytic activity of pooled microsomes from human (A), mouse (B) or rat (C) livers

Microsomes $(15 \mu \mathrm{g})$ from human liver $(\mathrm{n}=14)$, mouse liver $(\mathrm{n}=4)$ or rat liver $(\mathrm{n}=4)$ were pre-incubated in a total volume of $10 \mu \mathrm{l}$ with orlistat at various concentrations $(0,0.1$ or $1 \mu \mathrm{M}$ ) for $30 \mathrm{~min}$. To the pre-incubated mix, $2.5 \mu \mathrm{l}$ sample loading buffer was added. The samples were then subjected to native gel electrophoresis and stained for esterase activity with 1-naphythylacetate as described in the section of Materials and Methods. The staining intensity was captured by Carestream 2200 PRO Imager. This experiment was repeated four times. 
A

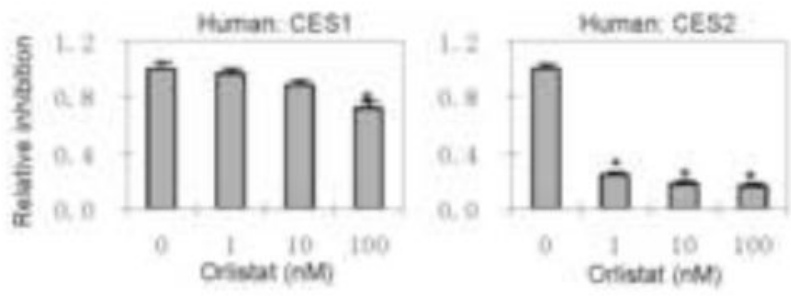

B

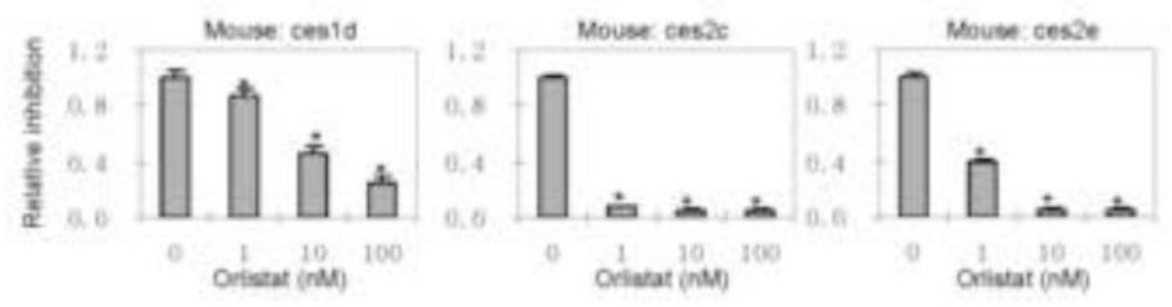

C
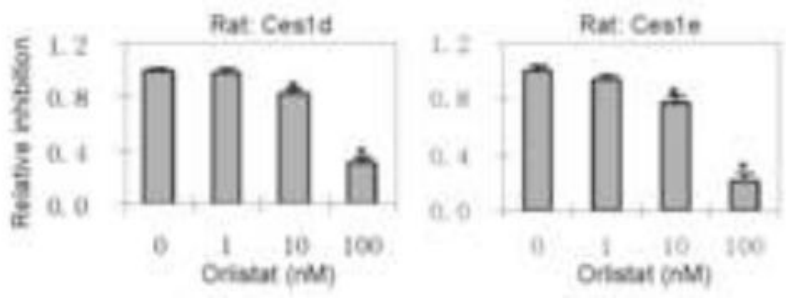

Fig. 3. Inhibition of $p$-nitrophenylacetate hydrolysis of recombinant carboxylesterases from human (A), mouse (B) or rat (C) livers by orlistat

Cell lysates $(1-5 \mu \mathrm{g})$ from $293 \mathrm{~T}$ cells transfected with a carboxylesterases were preincubated in a total volume of $10 \mu \mathrm{l}$ with orlistat at various concentrations $(0-100 \mathrm{nM})$ for $30 \mathrm{~min}$. The hydrolysis of $p$-nitrophenylacetate was determined as described above. Results were from three separate experiments. An asterisk sign indicates statistical significance from the control $(\mathrm{P}<0.05)$. 
A

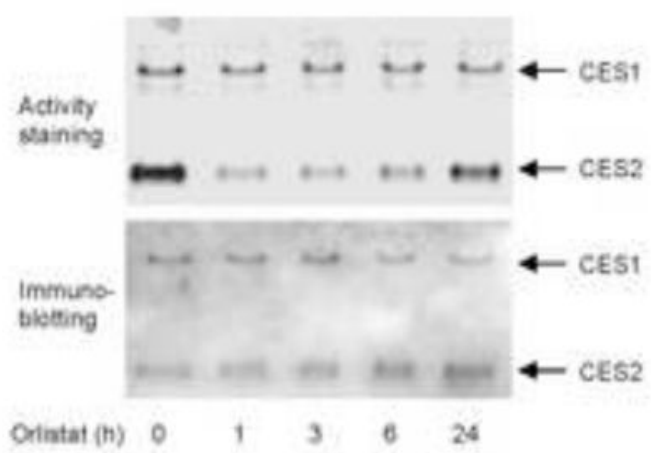

B

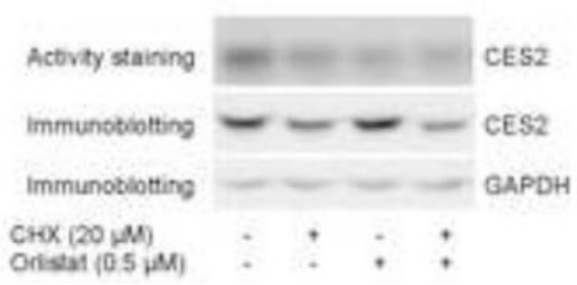

Fig. 4. Intracellular inhibition of CES1 by orlistat as a function of incubation time and CHX (A) Inhibition as a function of incubation time LS180 cells $\left(2 \times 10^{5}\right)$ were seeded in 12-well plates overnight. The cells were then treated with orlistat $(0.5 \mu \mathrm{M})$ for 1, 3, 6 and $24 \mathrm{~h}$. Cells were harvested and lysates $(10 \mu \mathrm{g})$ were analyzed by native-gel electrophoresis stained for esterase activity with 1-naphythylacetate as descried in the section of Materials and Methods. The same gel was analyzed by Western blotting with mixed antibodies against CES1 and CES2. (B) Inhibition in the presence or absence of CHX LS180 cells $\left(2 \times 10^{5}\right)$ were cultured as above and treated for $24 \mathrm{~h}$ with orlistat $(0.5 \mu \mathrm{M})$, CHX $(20 \mu \mathrm{M})$ or both. Cell lysates $(10 \mu \mathrm{g})$ were analyzed for esterase activity by native gel electrophoresis (CES2) or Western blotting (CES2 and GAPDH). All experiments were repeated three times. 
A

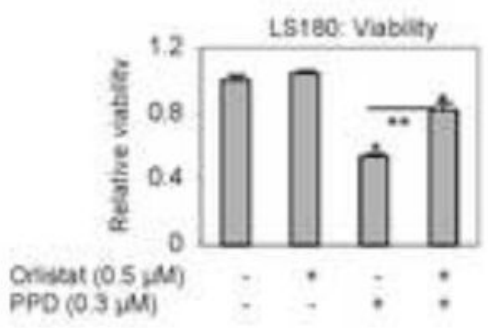

B

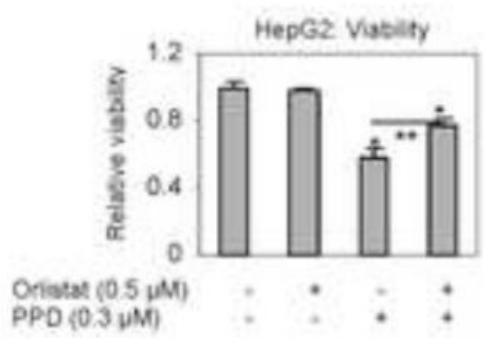

C

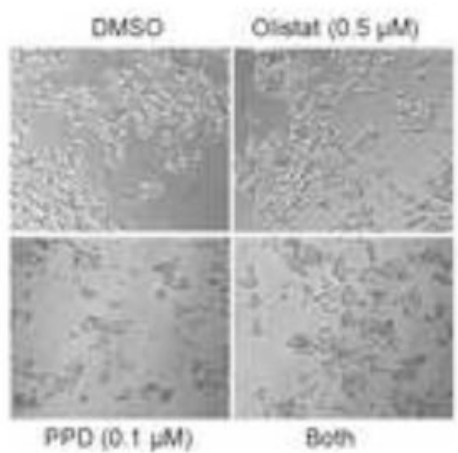

Fig. 5. Decreased cell killing of PPD by orlistat

(A) Decreased cell killing of PPD by orlistat in LS180 tumor line LS180 cells were plated in 96- or 12-well plates at a confluence of $30 \%$. Cells were then treated with orlistat $(0.5 \mu \mathrm{M})$, PPD $(0.3 \mu \mathrm{M})$ or both. After $48 \mathrm{~h}$-culture, cells were analyzed by MTT. Single or double asterisk signs indicate statistical significance from the control ( $\mathrm{P}<0.05)$. (B) Decreased cell killing of PPD by orlistat in HepG2 tumor line HepG2 cells were cultured and treated as LS180 cells, so were the determinations on cell viability. Single or double asterisk signs indicate statistical significance from the control $(\mathrm{P}<0.05)$. (C) Microscopic images of cells of LS180 $(100 \times)$. 
A

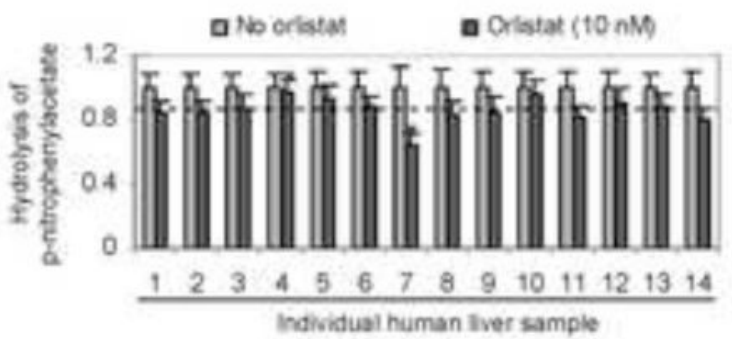

B

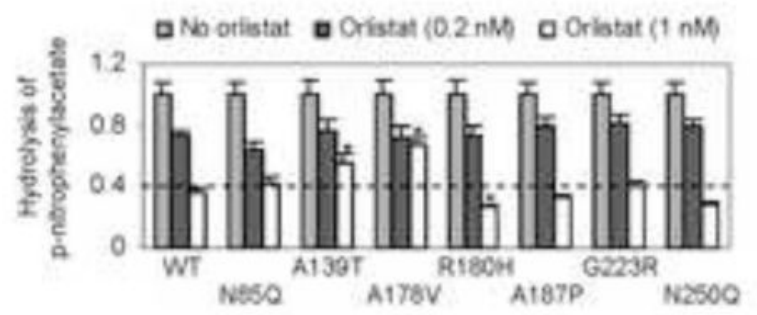

C Orlistat $\frac{\text { Activey }}{0.01,9} \frac{\text { Western }}{0.01,9 M}$ Wid type A.39t Atrov R180H
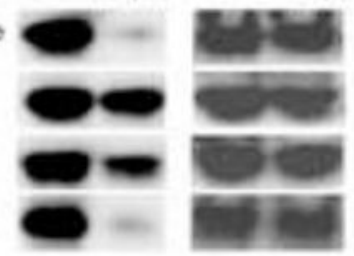

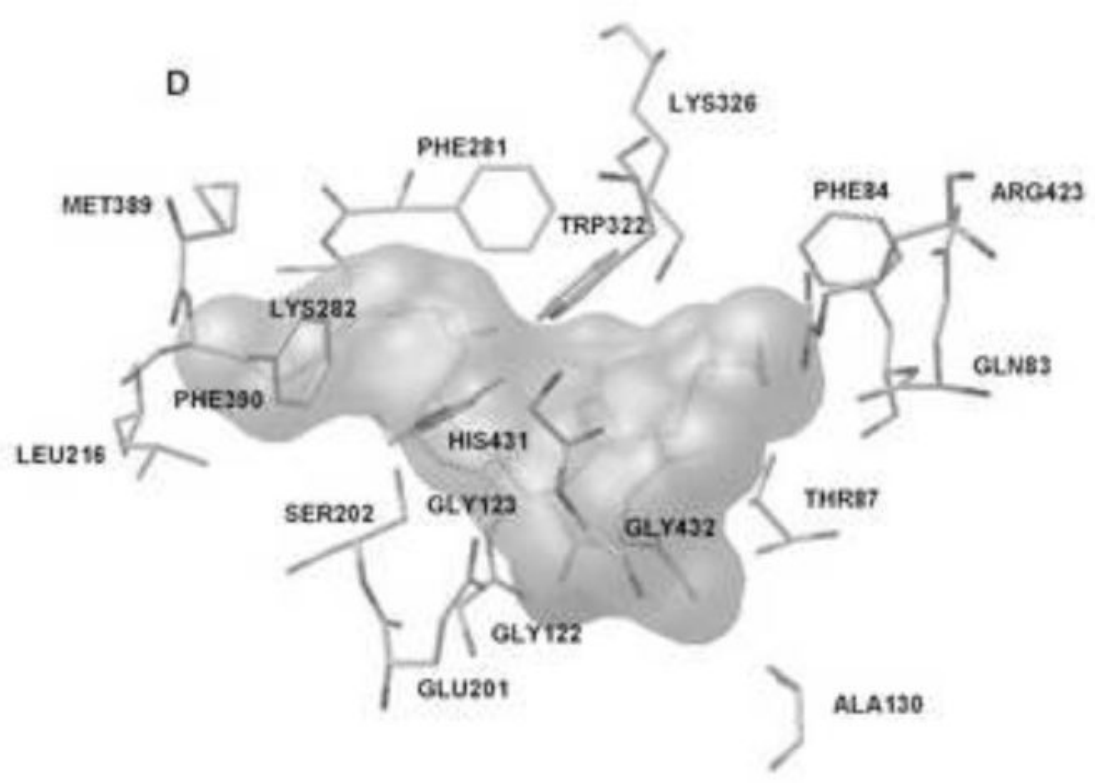

Fig. 6. Orlistat-inhibition of hydrolysis by individual liver samples and CES2 variants and molecular docking (A) Inhibition of hydrolysis of individual liver samples Microsomes (40 $\mu \mathrm{g})$ from individual human liver were pre-incubated in a total volume of $990 \mu \mathrm{l}$ with orlistat at various concentrations (10 $\mathrm{nM}$ ) for $30 \mathrm{~min}$. The hydrolytic activity was determined as described above. Results were from triplication. The dotted line indicates the average inhibition and an asterisk sign indicates statistical significance from the average inhibition $(\mathrm{P}<0.05)$. $(B)$ Inhibition of hydrolysis of CES2 variants Lysates $(5 \mu \mathrm{g})$ from cells transfected with CES2 (wild type or a variant) were pre-incubated in a total volume of $990 \mu \mathrm{l}$ with orlistat at 
various concentrations $(0-10 \mathrm{nM})$ for $30 \mathrm{~min}$. The hydrolytic activity was determined as described above. Results were from triplication. The dotted line indicates the average inhibition and an asterisk signs indicates statistical significance from the average inhibition. (C) Confirmation of differential inhibition by electrophoresis Cell lysates $(10 \mu \mathrm{g})$ were analyzed for esterase activity by native gel electrophoresis (CES2) or Western blotting (CES2 and GAPDH). All experiments were repeated three times. (D) Molecular docking of orlistat into CES2. 
A
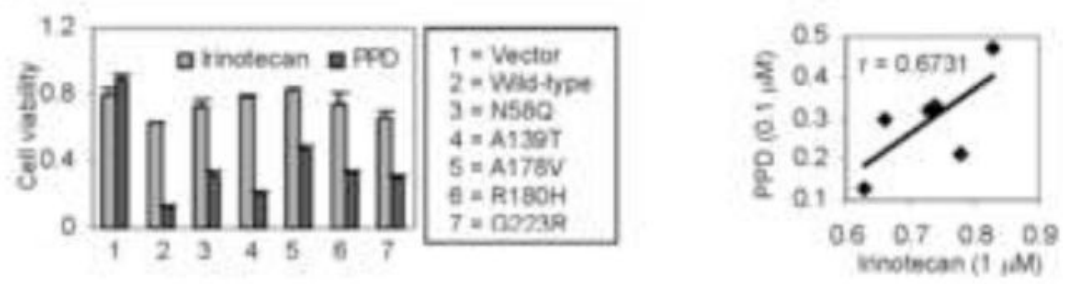

B
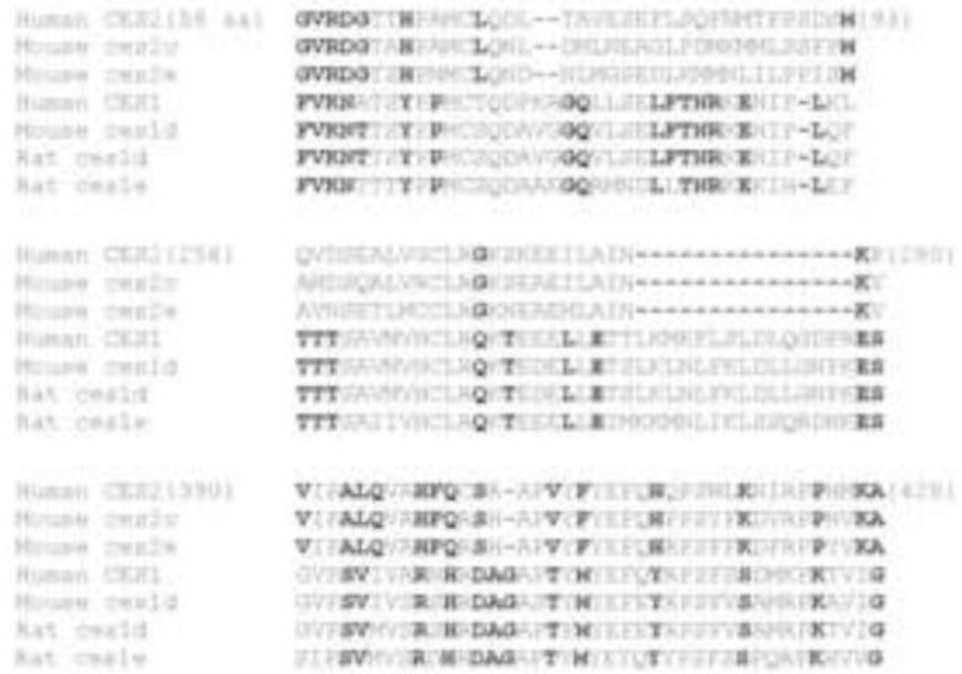

Fig. 7. Hydrolytic activation of prodrugs and sequence alignment analysis (A) Hydrolytic activation of irinotecan and PPD Cells (293T) were transfected with CES2 (wild-type or a mutant) or the vector. After overnight, the transfected cells were treated with DMSO, irinotecan $(1 \mu \mathrm{M})$ or PPD $(0.1 \mu \mathrm{M})$. The treatment lasted for $48 \mathrm{~h}$ and cell viability was determined. The relative viability between PPD and irinotecan treatment was analyzed by correlation. (B) Sequence alignment of human CES2 (numbered), mouse ces2c, mouse ces2e, human CES1, mouse ces1d, rat ces1d and ces1e. 
Table I

Sequences of primers for site-directed mutagenesis

\begin{tabular}{ll}
\hline Primer & Sequence \\
\hline CES2-N85Q & 5'-TTTCTTAGCCAGTTCCAGATGACCTTCCCTTCC-3' \\
CES2-A139T & 5'-GATGGTTCCATGCTGACTGCCTTGGAGAACGTG-3' \\
CES2-A178V & 5'-TACCTGGACCAAGTGGTTGCACTACGCTGGGTC-3' \\
CES2-R180H & 5'-CAAGTGGCTGCACTACACTGGGTCCAGCAGAAT-3' \\
CES2-A187P & 5'-GTCCAGCAGAATATCCCCCACTTTGGAGGCAAC-3' \\
CES2-G223R & 5'-CAAGGACTCTTCCACAGAGCCATCATGGAGAGT-3' \\
CES2-N250Q & 5'-TCCACGGTGGTGGCCCAACTGTCTGCCTGTGAC-3' \\
\hline
\end{tabular}

